\title{
The Dosimetric Characteristics and Potential Limitation in Clinical Application of a Low Energy Photon Intra-0perative Radiotherapy System
}

\author{
Zhenhua Xiao*, Ouyang Bin*, Zhenyu Wang, Botian Huang, Bixiu Wen" \\ Department of Radiation Oncology, The First Affiliated Hospital of Sun Yat-sen University, Guangzhou, China \\ Email: "wenbix@mail.sysu.edu.cn
}

Received 22 April 2015; accepted 23 May 2015; published 27 May 2015

Copyright (C) 2015 by authors and Scientific Research Publishing Inc.

This work is licensed under the Creative Commons Attribution International License (CC BY). http://creativecommons.org/licenses/by/4.0/

(c) (i) Open Access

\begin{abstract}
Purpose: To investigate the dosimetric characteristics of a low energy photon intra-operative radiotherapy (IORT) system and explore its potential limitation in clinical application. Methods: A special water phantom, a parallel-plate ionization chamber and an electrometer were used to measure the depth dose rate, isotropy of dose distribution in $\mathrm{X} / \mathrm{Y}$ plane, dosimetry reproducibility of bare probe and spherical applicators of different size which were used in comparison with the system data. Results: The difference in depth dose rate between the measurement and system data for bare probe is $-2.16 \% \pm 1.36 \%$, the range of the relative deviation for isotropy in the $X / Y$ plane is between $-1.9 \%$ and $2.1 \%$. The difference in depth dose rate, transfer coefficient, isotropy in $X / Y$ plane between the measurement and system data for the whole set of spherical applicators is $-10.0 \%-2.3 \%,-8.9 \%-4.2 \%$ and $-1.6 \%-2.6 \%$, respectively. Higher surface dose rate and steeper gradient depth dose are observed in smaller spherical applicators. The depth dose rate and isotropy for bare probe and spherical applicators have been shown good reproducibility. The uncertainty of measurement is associated with the positioning accuracy, energy response, noise current and correction function $f^{\prime}(R)$. Conclusions: Thorough commissioning of the low energy photon IORT system helps us better understand the dosimetry characteristics, verify the system data, obtain adequate data for clinical application and routine quality assurance. The steep gradient depth dose and limited treatment range may restrain its potential in clinical application.
\end{abstract}

\section{Keywords}

Intra-Operative Radiotherapy, Low Energy Photon, Dosimetry, Isotropy, Limitation

\footnotetext{
"The first two authors contribute equally.

\#Corresponding author.
}

How to cite this paper: Xiao, Z.H., et al. (2015) The Dosimetric Characteristics and Potential Limitation in Clinical Application of a Low Energy Photon Intra-Operative Radiotherapy System. International Journal of Medical Physics, Clinical Engineering and Radiation Oncology, 4, 184-195. http://dx.doi.org/10.4236/ijmpcero.2015.42023 


\section{Introduction}

Intra-operative radiotherapy (IORT) delivers single high dose radiation directly to the tumor bed within a relative short period of treatment time during the surgical operation, which requires a higher surface dose to protect the deep normal tissues. It often uses high energy electron beam or low energy photon beam. Modern IORT is usually delivered in the operating room, which requires that the device is light weighted and easy to move with high quality of radiation protection. Dedicated IORT device commercially available includes Mobetron ${ }^{\circledR} \mathrm{MeV}$ electron beam system [1] (Intra Op Medical Corporation, California, USA), NOVAC ${ }^{\text {TM }} 7$ system (New Radiant Technology SpA, Italy) and the INTRABEAM ${ }^{\circledR} 50$ kV X-ray device (Carl Zeiss Medical Company, Germany).

Since the results from the randomized TARGIT A trial were published, IORT has been applied for locally advanced or recurrent rectal cancer [2], superficial cutaneous malignancies [3] and as full dose partial breast irradiation (PBI) or as a boost after whole-breast radiatherapy (WBI) in early stage breast cancer [4] [5]. Vaidya J.S. et al. have analysis overall survival of using as single-dose targeted intraoperative radiotherpay (TARGIT) versus fractionated external beam radiotherapy (EBRT) for breast cancer. For patients enrolled at 33 centers in 11 countries, 1721 patients were randomised to TARGIT and 1730 to EBRT. The 5-year risk for local recurrence in the conserved breast was 3.3\% (95\% CI 2.1 - 5.1) for TARGIT versus $1.3 \%(0.7-2.5)$ for EBRT ( $\mathrm{p}=$ 0.042). Wound-related complications were much the same between groups but grade 3 or 4 skin complications were significantly reduced with TARGIT (four of 1720 vs 13 of $1731, \mathrm{p}=0.029$ ) [6]. TARGIT-B (for boost, ISRCTN43138042) is an ongoing multicenter randomised controlled trial that began in 2013, which is testing the replacement of EBRT boost to the tumor bed by a TARGIT boost given during surgery. An ongoing open registry study, TARGIT-R (for registry, ISRCTN91179875), began in 2013, aiming to monitor the long-term effectiveness and safety of the patients treated with TARGIT following breast conserving surgery for early breast cancer [7].

Eaton D.J. et al. described the dosimetry of the INTRABEAM ${ }^{\circledR}$ system with the spherical applicators [8]. Maxime Goubert et al. have reported dosimetric behavior of the system equipped with flat and surface applicators [3]. There still remains controversary for the dosimetric characteristics and clinical application of IORT. In this paper we introduce the commissioning test of the INTRABEAM ${ }^{\circledR} 50 \mathrm{kV}$ x-ray system by investigating the dosimetric characteristics of the low energy photon IORT system and explore its potential limitation in clinical application.

\section{Method and Materials}

\subsection{Introduction of the System}

The components of INTRABEAM system mainly include the X-ray source (XRS) with internal radiation monitor (IRM), PRS 500 control console, user terminal for configuration and operation of the core system. The system is equipped with accessory device including quality assurance (QA) tools, source applicators of different shape and size. Radiation-shielding operation room is specially built for the $\mathrm{kV}$ energy level, $\mathrm{X}$-ray source device of IORT system.

The XRS, a micro X-ray generator with weight of only $1.5 \mathrm{~kg}$, is fixed into the six-degree freedom arm frame during clinical use. It can be locked and placed into the operation cavity from different angles within the large scope of the movement. The principle of XRS is similar to ordinary X-ray machine with an electron emitting cathode gun. After being accelerated in high voltage electric field, the high speed electron beams is drifted into a $100 \mathrm{~mm}$ long hollow probe with diameter of $3.2 \mathrm{~mm}$ to the gold target under the guidance of bending magnet. A spherical dose distribution is generated by bombard between the electrons and gold target in a deflection scanning way. The high voltage electric field can provide two modes $(40 \mathrm{kV}$ and $50 \mathrm{kV})$ accelerating voltage. The 40 $\mathrm{kV}$ mode provides a single tube current of $40 \mu \mathrm{A}$, whereas the $50 \mathrm{kV}$ mode provides $5,10,20,40 \mu \mathrm{A}$ tube current. This test was performed under $50 \mathrm{kV} / 40 \mu \mathrm{A} X$-ray.

Due to the low beam energy, dose falls rapidly (approximately to $1 / \mathrm{r} 3, \mathrm{r}$ is the distance between the interest point and the center of the source) in water [9], which led to high dose at the surface of the applicators while deep tissue was well protected. The INTRABEAM system is equipped with spherical, needle, flat and surface applicators which can theoretically be applied according to the position of interest and shape of tumor bed. Spherical applicators, a total number of 8 with $1.5 \mathrm{~cm}$ to $5.0 \mathrm{~cm}$ in diameter with $0.5 \mathrm{~cm}$ increment, are mainly used for the treatment of breast cancer [6]. Applicators consist of solid homogeneous polyether imide, which can 
be divided into two groups according to the structure mounted interiorly; the interior wall of the probe is embedded with a thin metal sheath for the applicators of diameter $\leq 3 \mathrm{~cm}$ whereas no metal sheath for those of diameter $>3 \mathrm{~cm}$ [8]. Applicator size is decided according to tumor size and the diameter of surgical cavity where those of $4.0-5.0 \mathrm{~cm}$ in diameters are relatively commonly used. Applicator is placed in surgical cavity before irradiation. Radiation oncologists should ensure the applicator and tumor cavity joint closely. Adjacent normal tissue can be protected with radiation protective spacer. Irradiation time is determined according to the size of applicator, prescription dose and treatment depth, which ranges from a few to over forty minutes.

\subsection{Dosimetry Measurement}

The device for measurement includes a dedicated water tank (see in Figure 1) with radiation protection design (Carl Zeiss Surgical GmbH), a parallel plate ionization chamber (volume: $0.0053 \mathrm{~cm}^{3}$, type 34013, PTW, Freiburg, Germany) and a dosimeter (UNIDOS E, PTW). Two waterproof measuring chambers in the water tank were designed to measure depth dose rate and isotropy respectively. The ionization chamber(IC) is inserted with the ionization holder into the measuring chamber, which is closed with cover during measurement. " $d_{I C}$ " is designated as "distance between the entrance foil and chamber top" in PTW calibration certificate. The thickness of measuring chamber was printed in the user manual as " $\mathrm{d}_{\mathrm{H}}$ ”. The air gap " $\mathrm{d}_{\mathrm{A}}$ ” between the upper surface of ionization chamber housing and the inside of measuring chamber wall is constantly $0.5 \mathrm{~mm}$. Those distances are considered in measuring the depth dose rate at particular position. The XRS is fixed into the platform which can be adjusted vertically with range of $10 \mathrm{~cm}$ and precision of $0.001 \mathrm{~mm}$. The lowest surface of the probe was defined as the isocenter of $0 \mathrm{~mm}$ in depth. Due to the certain thickness of the holder wall of the ionization chamber, the measurement in $\mathrm{Z}$ direction ranges from $2.0 \mathrm{~mm}$ to $44.0 \mathrm{~mm}$ with $0.5 \mathrm{~mm}$ increment when the distance is less than $40.0 \mathrm{~mm}, 1.0 \mathrm{~mm}$ for $40.0 \mathrm{~mm}$ or higher with a period of 1 minute for each point of measurement. The platform of the water tank is also designed with a turntable structure in the $\mathrm{X} / \mathrm{Y}$ plane for measuring the isotropic dose distribution. The XRS can be rotated with the platform every $45^{\circ}$ to the total of 8 angles (initial position was defined as $0^{\circ}$ during measurements). The $\mathrm{X}, \mathrm{Y}$ directions can be located by fine tune to align the central axis of the bare source probe with the center of the ionization chamber. The position of X, Y directions were kept unchanged for isotropic measurement.

The measurement of depth dose rate was made for bare probe (no applicator) and whole set of spherical applicators in Z direction, isotropy in $\mathrm{X} / \mathrm{Y}$ plane for bare probe. The repeatability of measurement was made for the bare probe and a spherical applicator of $\phi 4.5 \mathrm{~cm}$; transfer coefficient was calculated according to the function provided by the company and the transfer coefficient deviation was used to compare the difference between the system data and measurements. The data were analyzed and plotted by Matlab (MathWorks, USA) software. The depth dose rate $D_{w}$ at reference depth (r) were calculated by the Formula (1):

$$
\mathrm{D}_{\mathrm{w}}(\mathrm{r})[\mathrm{Gy} / \mathrm{min}]=\mathrm{N}_{\mathrm{k}}[\mathrm{Gy} / \mathrm{C}] \times \mathrm{Q}(\mathrm{r})[\mathrm{C}] \times \mathrm{T}[\mathrm{K}] / \mathrm{T}_{0}[\mathrm{~K}] \times \mathrm{P}_{0}[\mathrm{hPa}] / \mathrm{P}[\mathrm{hPa}] \times \mathrm{k}_{\mathrm{Q}} \times \mathrm{k}_{\mathrm{Ak} \rightarrow \mathrm{DW}} \times 1[1 / \mathrm{min}]
$$

$\mathrm{N}_{\mathrm{k}}$ = detector calibration factor (see the certificate, the number is 4247000000).

$\mathrm{Q}(\mathrm{r})=$ measured charge $\mathrm{Q}$ in one minute.

$\mathrm{T}=$ current temperature.

$\mathrm{T}_{0}=$ reference temperature (see calibration certificate of ionization chamber).

$\mathrm{P}_{0}=$ reference air pressure (see calibration certificate of ionization chamber).

$\mathrm{P}=$ current air pressure.

$\mathrm{k}_{\mathrm{Q}}$ = beam quality correction factor (see PTW certificate, the quality level of XRS is approximately T30).

$\mathrm{k}_{\mathrm{Ak} \rightarrow \mathrm{DW}}=$ air kerma $\rightarrow$ absorbed dose to water conversion (correction factor for PTW type 34013 IC inoization chamber, the number for T30 is 1.054).

The transfer coefficient $\mathrm{TF}_{\mathrm{z}}$ (spherical) at reference depth (r) were calculated by the Formula (2):

$$
\left(\mathrm{TF}_{\mathrm{z}}(\mathrm{r})\right)^{\text {spherical }}=\left(\mathrm{D}_{\mathrm{w}}(\mathrm{r})\right)^{\text {spherical }} /\left(\mathrm{D}_{\mathrm{w}}(\mathrm{r})\right)^{\mathrm{xrs}}
$$

$\left(D_{w}(r)\right)^{\text {spherical }}=$ calculated dose rate at reference depth $(r)$ according to Formula (1).

$\left(D_{w}(r)\right)^{\mathrm{xrs}}=$ calculated dose rate at the same position of $\mathrm{Z}$ direction without the spherical applicator.

The percentage error $E$ at reference depth (r) was defined by the Formula (3):

$$
E(r)=\left(\left(D_{w}(r)^{\text {measurement }}-D_{w}(r)^{\text {software }}\right) / D_{w}(r)^{\text {measurement }}\right) \times 100 \%
$$


$\mathrm{D}_{\mathrm{w}}(\mathrm{r})^{\text {measurement }}=$ dose rate at reference (r) calculated by Formula (1) according to charge $\mathrm{Q}$ read in the dosimeter.

$D_{w}(r)^{\text {softeware }}=$ dose rate at reference $(r)$ aquired from user manual or software profile in the control system.

\section{Results}

\subsection{Depth Dose Rate of Bare Probe}

The depth dose rate for the bare probe was measured three times. Figure 2 depicts the dose rate in Gy/min or error of measurement in percentage as a function of distance between the surface of XRS probe and the ionization chamber monitor-node. The red line represents the average measurement for three times, the data in blue line are obtained from the operation system and the repeatability of the measurements for 3 times are shown in percentage of error in black line. As shown in Figure 2(a) \& Figure 2(b), the maximum deviation of three times measurement for bare probe ranges from $0.14 \%$ to $1.3 \%$. The value of dose rate measured is higher than that obtained from the operation system when the distance between the surface of XRS probe to the ionization chamber monitor-node is $<5.0 \mathrm{~mm}$; the value measured is lower than that from the operation system when the distance is $10.0 \mathrm{~mm}$ or more. The average error between the actual measurement and the system is $-2.16 \% \pm$ $1.36 \%$ ranging from $-3.65 \%$ to $2.83 \%$.

\subsection{Isotropy of Bare Probe}

Figure 3(a) depicts isotropic dose in $\mathrm{X} / \mathrm{Y}$ plane for bare probe, the value of each angle was normalized to the average of 8 measurements at the specific distance. The consistency for the value of each measurement angle is relatively good. As shown in Figure 3(b), the relative deviation of measurements for four times ranged from $-1.9 \%$ to $2.1 \%$. The deviation of measurement at $90^{\circ}(-1.9 \%)$ and $225^{\circ}(2.1 \%)$ were relatively larger. The tendency of the measuring error is consistent with good repeatability.

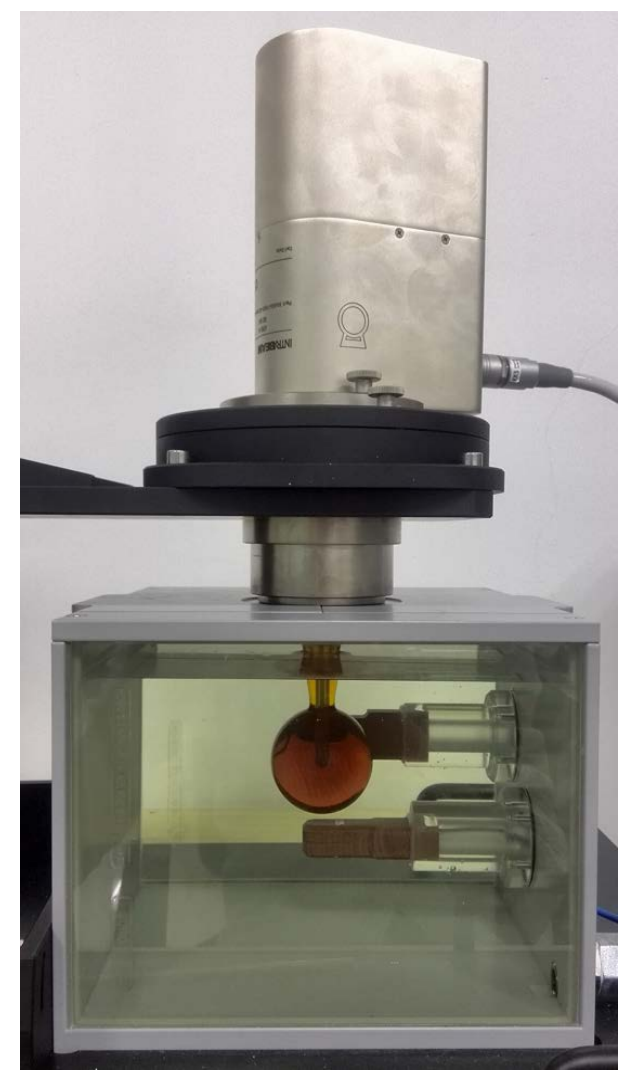

Figure 1. Schematic diagram of commissioning setup for a spherical applicator of $4.5 \mathrm{~cm}$ in diameter (water tank). 


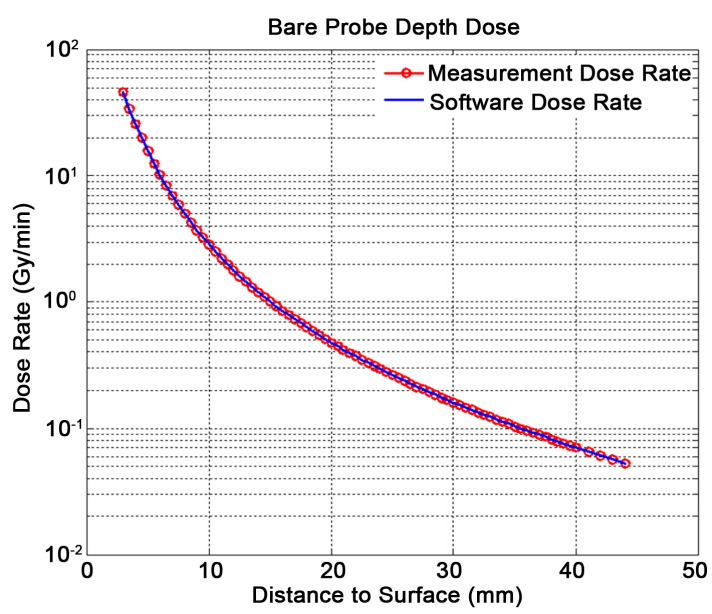

(a)

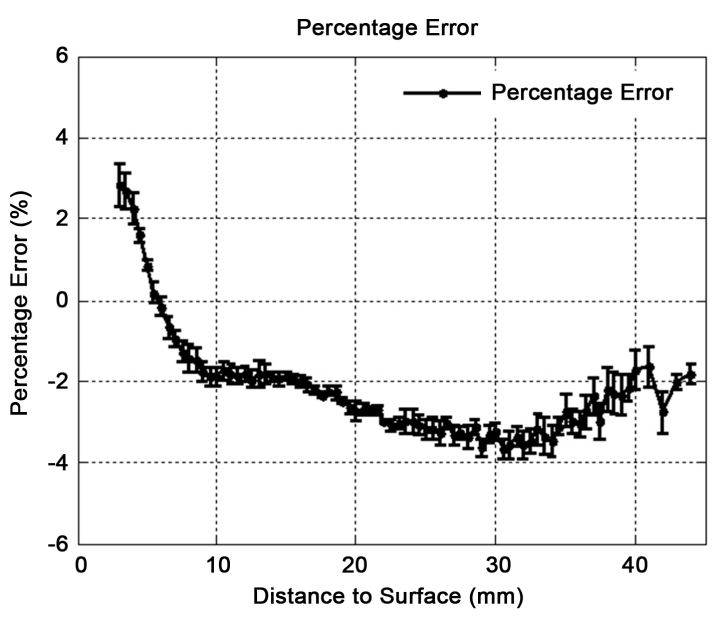

(b)

Figure 2. The depth dose rate (a) and the error in percentage; (b) measured in comparison with data from operation system for bare probe. The red line represents the average of measurement for three times, blue line depicts the data from the operation system, the black line for the deviation of measurement with bare probe in percentage error.

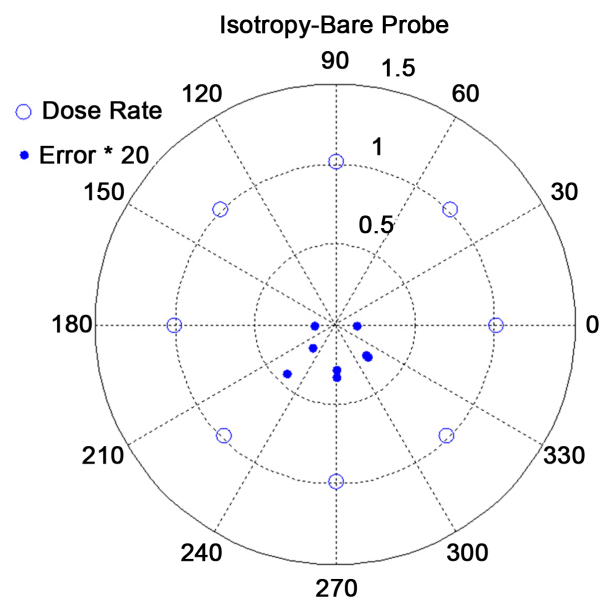

(a)

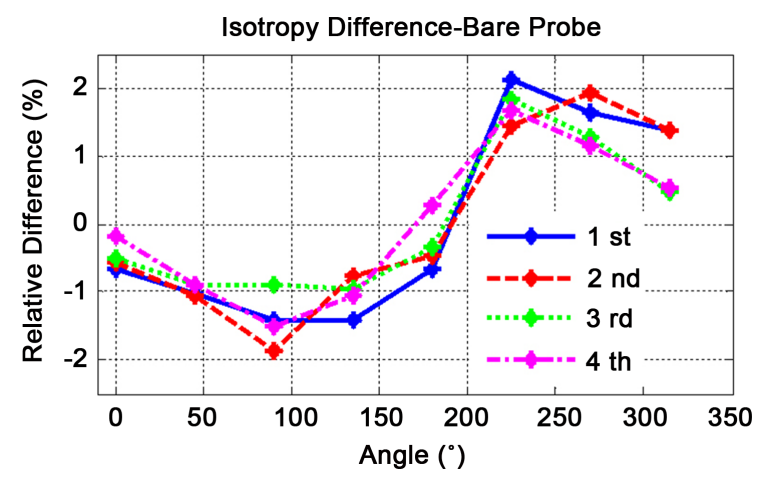

(b)

Figure 3. Isotrophy in $\mathrm{X} / \mathrm{Y}$ plane for bare probe. (a) Normalized value and relative deviation in 8 measurement angles. The hollow circle represents the normalized value, and solid dots the relative percentage deviation of each measurement point magnified 20 times; (b) Relative percentage deviation for 4 times measurement.

\subsection{Depth Dose Rate of Spherical Applicators}

Figure 4(a) depicts the curve of dose rate measured as a function of the distance from the surface of spherical applicators to ionization chamber monitor-node for 8 types of spherical applicators. The dose rate is the highest at the surface of the applicators which falls as the distance between the surfaces of spherical applicators to ionization chamber monitor-node increases. The dose rate varies significantly with the size of the applicator. For the applicator with smaller diameter the dose rate is higher with relative shorter treatment time if the same radiation dose is delivered. As the diameter of the applicator increases, the falling gradient in dose rate slows down. From Figure 4(a), we also observe that the curves of the dose rate overlaps for applicators in diameters $2.5 \mathrm{~cm}$ and 3.5 $\mathrm{cm}, 3.0 \mathrm{~cm}$ and $4.0 \mathrm{~cm}$, respectively.

Figure 4(b) shows the curve of depth dose rate as a function of distance between the isocenter of spherical applicator and ionization chamber monitor-node. Obvious separation of dose rate was observed. At the same distance from the isocenter, the dose rate is smaller in the applicators of diameter $\leq 3.0 \mathrm{~cm}$ than those of diameter $>3.0 \mathrm{~cm}$. The difference in depth dose rate, transfer coefficient and isotropy between measurements and system data is presented in Table 1 . The average deviation in depth dose rate between measurements and system 


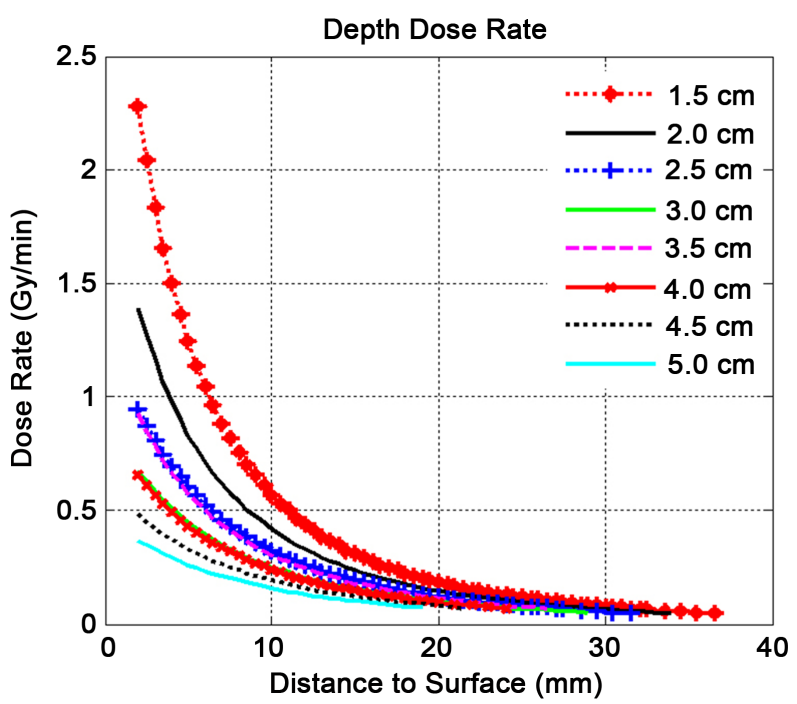

(a)

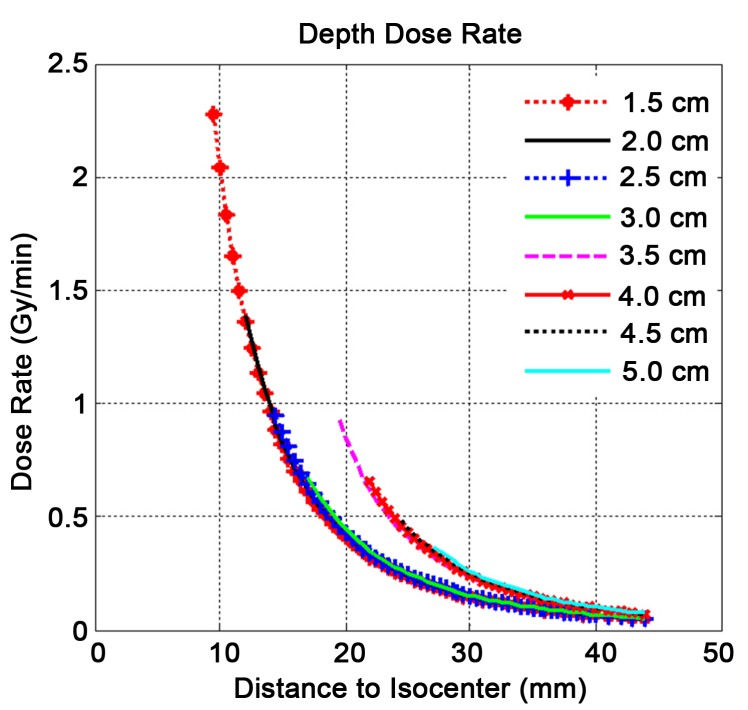

(b)

Figure 4. The curve of depth dose rate measured for each spherical applicator. (a) The curve of depth dose rate of different distance from the surface of spherical applicators to ionization chamber monitor-node; (b) The curve of depth dose rate of different distance from the isocenter of spherical applicator to ionization chamber monitor-node.

Table 1. The difference in depth dose rate, transfer coefficient and isotropy for spherical applicators.

\begin{tabular}{cccc}
\hline Diameters $(\mathrm{cm})$ & Deviation of depth dose rate (range) (\%) & Deviation of transfer coefficiency (range) (\%) & Isotropy (range) (\%) \\
\hline 1.5 & $-5.0 \pm 1.7(-10.0--2.4)$ & $-2.5 \pm 2.2(-8.9--0.2)$ & $0 \pm 1.2(-1.6-1.2)$ \\
2.0 & $-5.1 \pm 0.90(-7.9--3.5)$ & $-2.6 \pm 1.2(-6.6--1.4)$ & $0 \pm 1.0(-1.2-1.4)$ \\
2.5 & $-4.8 \pm 0.52(-6.3--3.4)$ & $-2.2 \pm 0.7(-4.7--1.3)$ & $0 \pm 1.1(-1.2-1.7)$ \\
3.0 & $-5.0 \pm 0.67(-6.5--3.0)$ & $-2.3 \pm 0.3(-3.0--1.7)$ & $0 \pm 1.2(-1.4-1.5)$ \\
3.5 & $-2.0 \pm 0.50(-2.8--0.3)$ & $0.8 \pm 0.5(-0.4-2.0)$ & $0 \pm 1.5(-1.5-1.8)$ \\
4.0 & $-1.5 \pm 1.1(-3.3-1.4)$ & $1.4 \pm 1.2(-0.9-4.2)$ & $0 \pm 1.4(-1.3-2.0)$ \\
4.5 & $-0.1 \pm 0.9(-1.4-2.3)$ & $1.5 \pm 0.9(0.3-3.7)$ & $0 \pm 1.4(-1.4-2.6)$ \\
5.0 & $-0.2 \pm 0.5(-1.1-1.1)$ & $2.4 \pm 0.6(1.5-4.0)$ & $0 \pm 1.1(-1.0-1.6)$ \\
\hline
\end{tabular}

data for the applicators of diameter $\leq 3.0 \mathrm{~cm}$ is in the range from $-5.1 \%$ to $-4.8 \%$; it reduces to $-2 \%--0.1 \%$ with the increase in diameter of the applicators.

\subsection{Transfer Coefficient of Spherical Applicators}

The transfer coefficient of spherical applicator is defined as ratio between the depth dose rates with or without applicator at the same distance to the source isocenter. The depth dose rate can be obtained by multiplication of the bare source dose rate and the conversion coefficient. Figure 5 is the curve for deviation in transfer coefficient between measured values and the system data for spherical applicators. The average deviation of transfer coefficient at the same distance between the isocenter of spherical applicator and ionization chamber monitor-node for applicators $\leq 3.0 \mathrm{~cm}$ ranges from $-2.6 \%$ to $-2.2 \%$; whereas the average deviation for tors $>3.0 \mathrm{~cm}$ is between $0.8 \%$ and $2.4 \%$. The more detailed data for difference in transfer coefficient between measurements and system data are presented in Table 1 for each individualized applicator.

\subsection{Isotropy of Spherical Applicator}

As shown in Table 1, the average deviation in isotropy between measurements and system data of different spherical applicator's $\mathrm{X} / \mathrm{Y}$ plane ranges from $-1.4 \%$ to $2.6 \%$ which shows no obvious change as the diameter of applicator increases. Figure 6(a) \& Figure 6(b) depicts the representative isotropy for dose distribution in X/Y plane for a spherical applicator of diameter $4.5 \mathrm{~cm}$ which shows the consistency of the measurement values. 


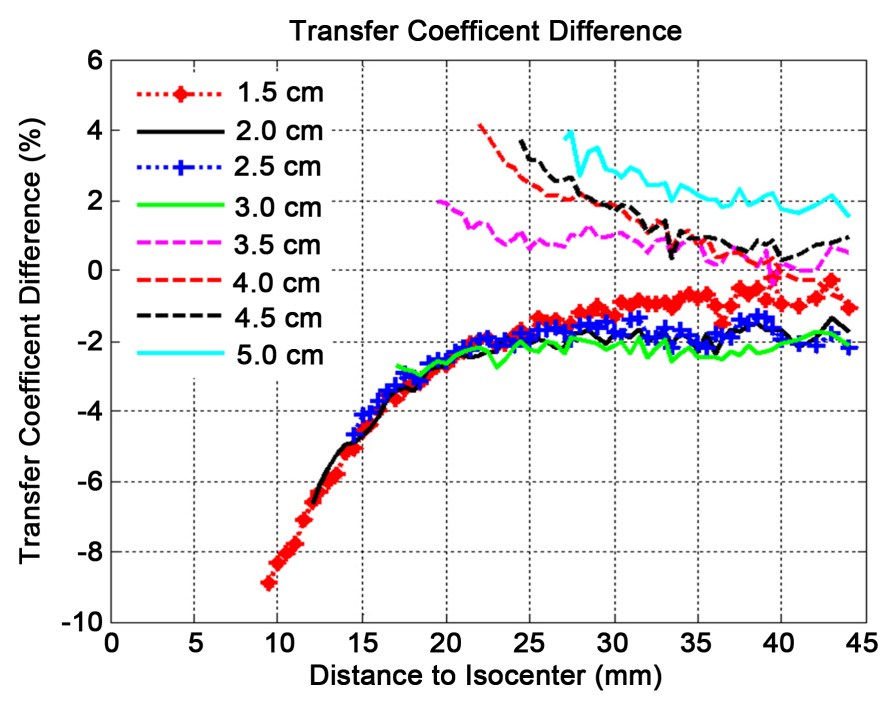

Figure 5. The curve for deviation in transfer coefficient between measured values and the system data for spherical applicators.

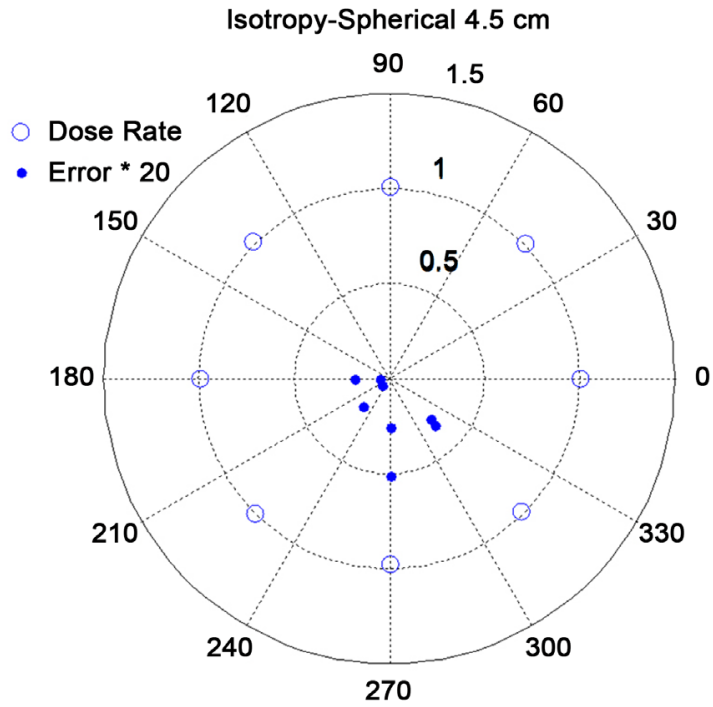

(a)

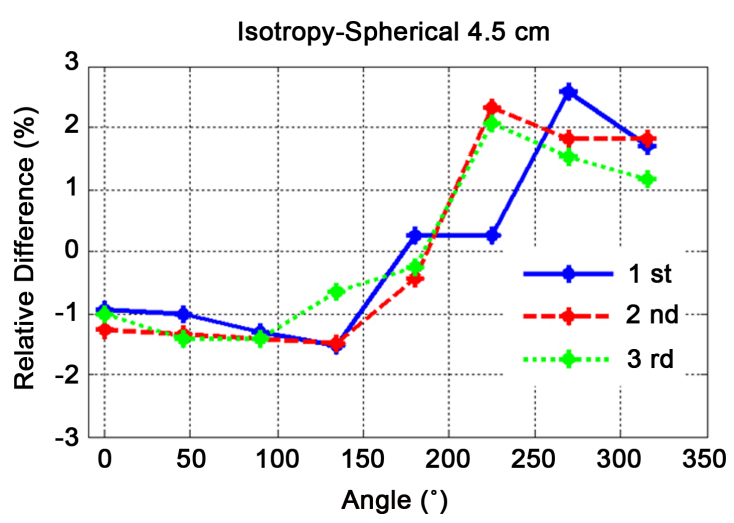

(b)

Figure 6. The representative isotropy in X/Y plane for a $\phi 4.5 \mathrm{~cm}$ spherical applicator. (a) Point view of normalized value and the relative deviation; (b) The percentage relative deviation of 3 times measurements.

\subsection{Repeatability of Spherical Applicator}

The Dose rate was measured in 3 times for $4.5 \mathrm{~cm}$ spherical applicator, the deviation in repeatability ranges from $0.2 \%$ to $0.7 \%$, which shows good repeatability and it gradually gets better as the depth increases. As shown in Figure 7(a) \& Figure 7(b), poorer repeatability and increased deviation of error are observed when the distance $>15 \mathrm{~mm}$ from the surface.

\subsection{Potential Limitation of Clinical Application}

Figure 8 depicts the graphic of dose distribution for the spherical applicator in diameter $4.5 \mathrm{~cm}$. The doses measured at the distance of $0.2,0.5,1.0$, and $2.0 \mathrm{~cm}$ are $15.3,10.7,6.4$ and $2.7 \mathrm{~Gy}$, respectively when surface dose of $20 \mathrm{~Gy}$ is prescribed. Table 2 lists the depth dose value for spherical applicators when the surface dose of $20 \mathrm{~Gy}$ is prescribed, which shows fast dose falling with gradual increasing depth. The doses range from 35.0\% 


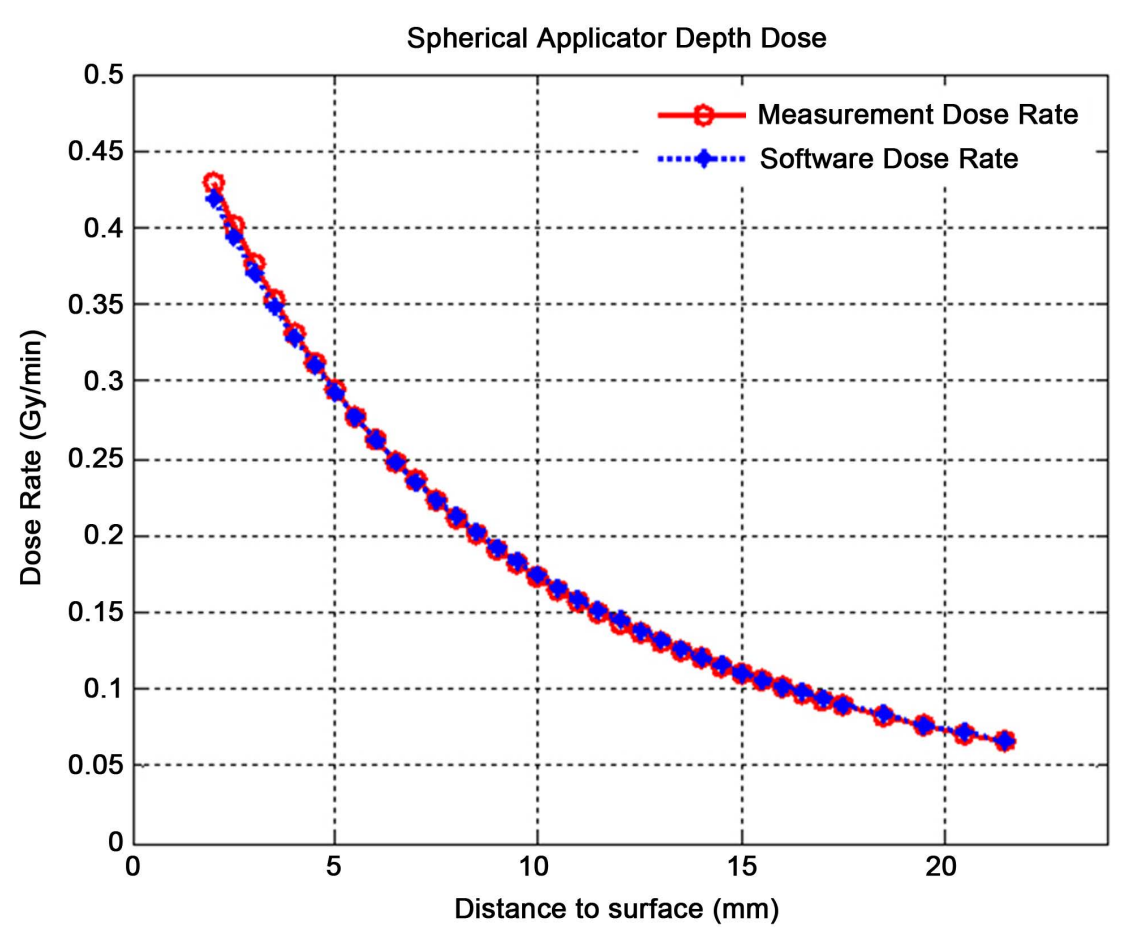

(a)

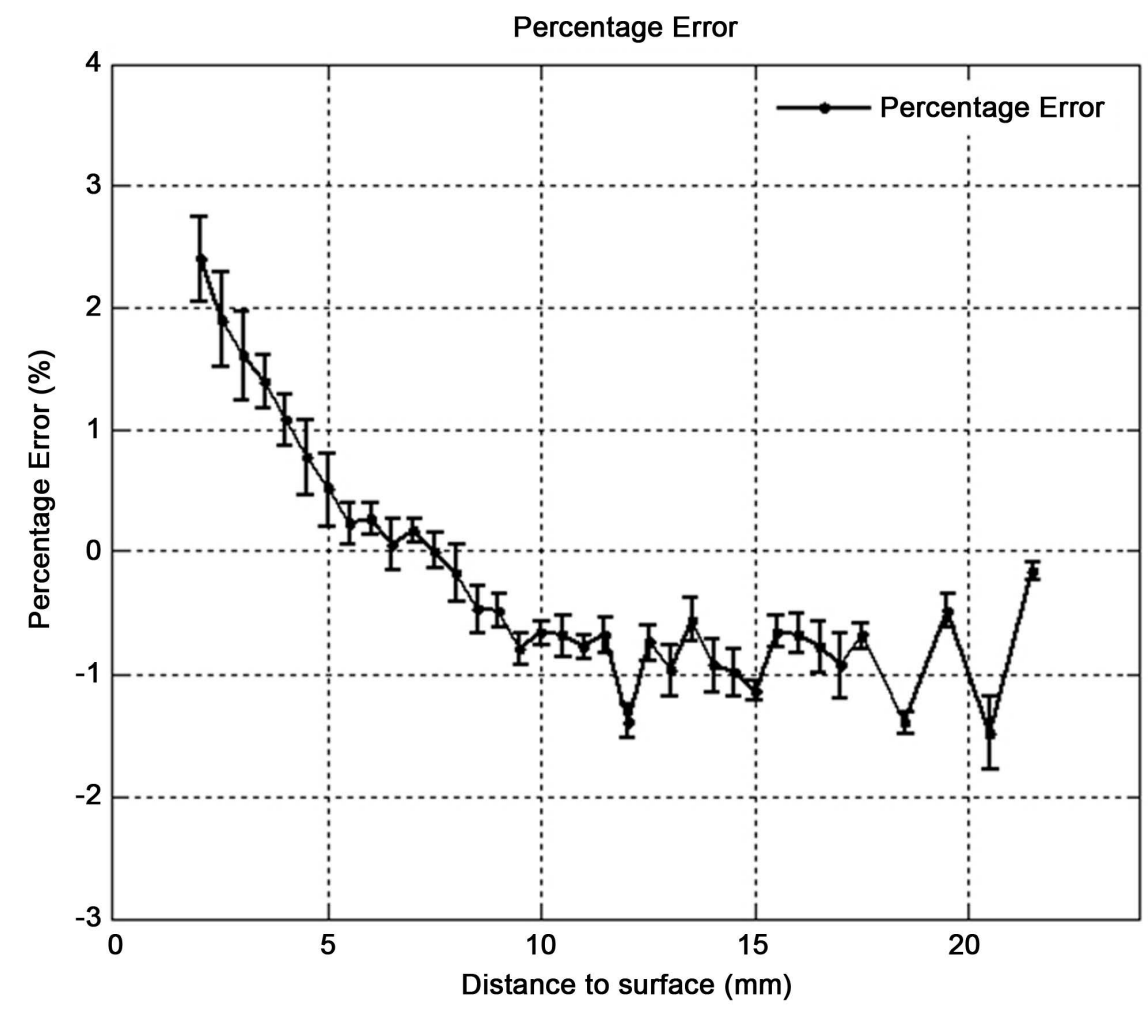

(b)

Figure 7. The curve for measurement value of depth dose rate and comparison chart with system data for $\phi 4.5 \mathrm{~cm}$ spherical applicator. (a) The depth dose rate for $\phi 4.5 \mathrm{~cm}$ spherical applicator. The red line intends for the average 3 measurement values, the blue line for the system value; (b)The error in percentage for the measurements in 3 times. 


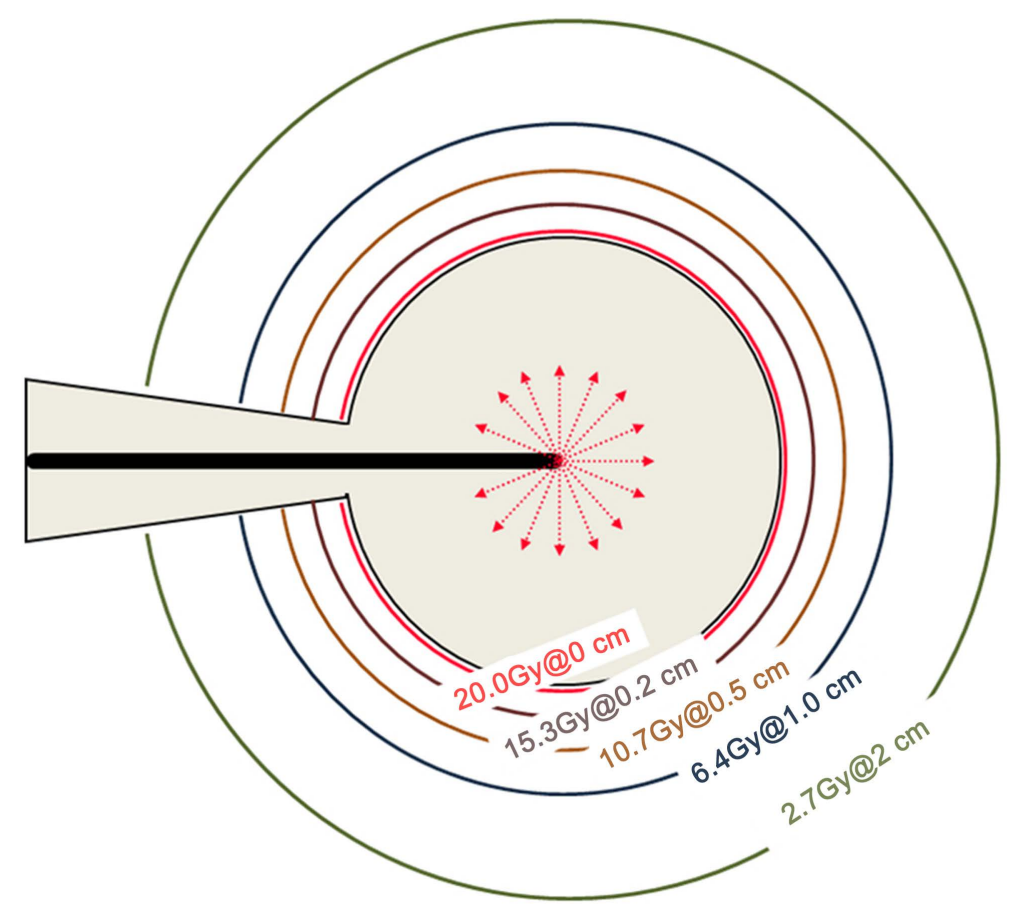

Figure 8. Dose distribution for a $\phi 4.5 \mathrm{~cm}$ spherical applicator when the surface dose of $20 \mathrm{~Gy}$ is prescribed.

Table 2. Depth dose rate (Gy) at serial typical depths for spherical applicators when surface dose of 20 Gy is prescribed.

\begin{tabular}{|c|c|c|c|c|c|c|}
\hline \multirow{2}{*}{ Diameter (cm) } & \multicolumn{6}{|c|}{ Distance from surface (mm) } \\
\hline & 0 & 2 & 5 & 10 & 15 & 20 \\
\hline 1.5 & 20.0 & 12.7 & 7.0 & 3.3 & 1.8 & 1.1 \\
\hline 2.0 & 20.0 & 13.5 & 8.1 & 4.1 & 2.4 & 1.5 \\
\hline 2.5 & 20.0 & 14.2 & 9.1 & 4.9 & 2.9 & 1.9 \\
\hline 3.0 & 20.0 & 14.8 & 10.0 & 5.7 & 3.5 & 2.3 \\
\hline 3.5 & 20.0 & 14.0 & 8.9 & 4.8 & 2.9 & 1.8 \\
\hline 4.0 & 20.0 & 14.7 & 9.9 & 5.6 & 3.5 & 2.3 \\
\hline 4.5 & 20.0 & 15.3 & 10.7 & 6.4 & 4.1 & 2.7 \\
\hline 5.0 & 20.0 & 15.6 & 11.2 & 6.8 & 4.5 & 3.1 \\
\hline
\end{tabular}

to $56.0 \%$ at $5 \mathrm{~mm}$ from the surface of the spherical applicators and fall to $16.5 \%-34.0 \%$ at $10 \mathrm{~mm}$ and $5.5 \%$ $15.5 \%$ at $20 \mathrm{~mm}$.

\section{Discussion}

Dose measurement tools used for INTRABEAM system include water tank/ionization chamber [10] [11], film/ solid water phantom [11], Thermo-luminescence [12], etc. Water tank/ionization chamber has the highest accuracyamong those instruments [13]. Schneider et al. have compared homogeneity of each dose distribution and depth-dose measurements for flat and surface applicators using film dosimetry in a solid water phantomand a soft X-ray ionization chamber in a water tank [10]. One of the important factors influencing the accuracy of the measurement is the relative position error of X-ray source to ionization chamber due to sharp attenuation of low energy X-ray in the water. The minimal position change will lead to relatively large measurement deviation. As 
shown in depth dose rate curve fitting function and differential coefficient data, the dose gradient at 3 and 10 $\mathrm{mm}$ from the source isocenteris $60 \% / \mathrm{mm}$ and $24 \% / \mathrm{mm}$, respectively. The position error of $\pm 0.1 \mathrm{~mm}$ will lead to deviation of dose rate at $\pm 6 \%$ and $\pm 2.4 \%$, respectively.

Eaton et al. have reported that the positioning of X-ray source is the most important factor that affects the measurement result and the measurement accuracy may be affected by the ionization chamber's volume effects in the area of steep dose falling, which may account for the poorer repeatability of measurement near the isocenter [13]. Other factors influence the accurate measurement including the energy response of ionization chamber [13], low voltage of X-ray [14], etc. The spectrum of X-ray approaching to the surface of the probe is complex ranging from 0 to $50 \mathrm{kV}$ with a large number of low energy $\mathrm{kV} \mathrm{X}$-ray [14] $(<20 \mathrm{kV})$ which showed rapid attenuation in water [15] resulting in significant difference at different depth and the measurement deviation could reach to $\pm 2.2 \%$ due to the energy response [9].

Another important reason for poor repeatability is that the measurement can be affected by noise current. The sensitive volume of ionization chamber is only $0.0053 \mathrm{~cm}^{3}$, and the ionization chamber is supposed to collect only approximately $25 \mathrm{pC}$ charge per minute at the depth of $35 \mathrm{~mm}$ during the measurement of X-ray source, which has led to low signal-to-noise ratio influenced by noise current. The uncertainty of measurement for ionization chamber could reach to $\pm 3.4 \%$ [9]. The accuracy and repeatability of measurement will gradually decrease with further increase in the distance from the X-ray source since ionization chamber collects less charge per minute (about $16.5 \mathrm{pC}$ ).

The type of ionization chamber, design of measuring chamber for water tank, method for calculation and condition of acquisition system for absorbed dose are different between users and company system. The calculation of absorbed doses, transfer coefficient and isotropy in $\mathrm{X} / \mathrm{Y}$ plane for the measurement data requires the manufacturer to provide the correction function $\mathrm{f}^{\prime}(\mathrm{R})$ for bare probes and spherical applicators of different size. The $\mathrm{f}^{\prime}(\mathrm{R})$ value is different at different depth which may introduce correction error, the error for uncertainty of $\mathrm{f}^{\prime}(\mathrm{R})$ is relatively larger when the depth is shallower. The average of uncertainty for $f^{\prime}(R)$ is $\pm 7.8 \%$.

Armoogum et al. analyzed the factors that affect the measurement result including temperature and atmospheric pressure correction factor, ionization chamber position deviation, ionization chamber current, the chamber/dosimeter calibration factor, output drift and calibration of absorbed dose [9]. Among these factors positional deviation is the most significant one. The estimation of total uncertainty for all these factors can reach to $\pm 10.8 \%$. Our data have shown that deviation between the measurement data and the system ranges from $-10.0 \%$ to $-5.0 \%$ for spherical applicator $\leq 3 \mathrm{~cm}$ in diameter and within $\pm 5.0 \%$ for the applicators $>3 \mathrm{~cm}$ in diameter, which are comparable with the data reported in literatures [9].

Our data have also shown a relative larger deviation of isotropy in $\mathrm{X} / \mathrm{Y}$ plane measured at $90^{\circ}(1.9 \%)$ and $225^{\circ}(2.1 \%)$. The possible reasons include: 1$)$ the probe is a hollow needle with $100 \mathrm{~mm}$ long and $3.2 \mathrm{~mm}$ outside diameter which may be bended during the operation with need to be calibrated before each use since system requirements of probe bending value is less than $0.1 \mathrm{~mm}$; 2) there may exist certain errors during the rotation of the water tank platform, which may lead to different distance from the tip of the probe to the ionization chamber monitor units in different angles; 3 ) the noise current may influence the correct measurements (the dosimeter charge is about $16.5 \mathrm{pC} / \mathrm{min}$ without applicators).

The clinical application of IORT with INTRABEAM system is determined by the size and category of applicators. The biggest diameter for spherical and tablet applicators is $5.0 \mathrm{~cm}$ and $6.0 \mathrm{~cm}$, respectively. The surrounding wall is embedded with thin metal sheath interiorly for spherical applicators of diameter $\leq 3 \mathrm{~cm}$. Low energy X-ray can be attenuated rapidly through the metal sheath. The gradient of dose rate value for spherical applicators in diameter $\leq 3 \mathrm{~cm}$ were larger than that for applicators with diameter $>3 \mathrm{~cm}$ when measured at the same distance from the isocenter.

Our results have shown that the steep dose gradient exists from $0 \mathrm{~cm}$, the surface of the applicator to $1.0 \mathrm{~cm}$; the higher surface dose rate and the greater dose gradient have been observed for the smaller applicators. When the region of treatment is too large, it will be very difficult to calculate accurate dose distribution due to lack of beam bridging technology with over- or under-dose. The limited region and depth of treatment may restrain from its potential in clinical application. Vaidya JS et al. have reported in TARGIT A, a phase III clinical trial that for patients with early breast cancer $<2 \mathrm{~cm}$ the tumor local control rate of IORT is not inferior to external beam radiotherapy after breast conserving surgery; external beam radiotherapy should be considered for patients with tumor of $2-3 \mathrm{~cm}$ or with poorer prognostic factors [6]. Sperk E et al. have reported that patient selection for IORT should be restrictive when provided as accelerated partial breast irradiation (APBI) [4]. 
The system only uses water for dose calculation whereas homogeneity of human structure cannot be revised. The calculated dose value and the deviation will be very big in different tissue due to the spectrum chacteristics of the low energy photon $(\leq 50 \mathrm{kV})$; the dose distribution will be influenced by the air gap between tissues and the applicator [14]. Monte Carlo modelling could be used in comparison with result from ionization chambers, radiochromic film and other dosimeters such as TLDs on the subsequent periodic QA tests for the INTRABEAM system [8]. There exist great difficulties for external beam radiotherapy when needed to supplement the postoperative radiotherapy especially for the accurate dose calculation and precise delineation of target and surrounding normal tissue irradiated especially for the nerves and blood vessels. All these factors mentioned above may seriously restrict INTRABEAM system to be widely used in clinical practice.

\section{Conclusion}

In summary, thorough commissioning of INTRABEAM system helps us better understand the dosimetry characteristics, verify the system data and a quire adequate data for clinical application and routine quality assurance. It is necessary to establish the benchmark for long term quality assurance based on the measurement data. The characteristics of high dose at the surface of applicator, great dose gradient and limited treatment range may restrain from its potential in wide clinical application.

\section{References}

[1] Beddar, A.S. (2005) Stability of a Mobile Electron Linear Accelerator System for Intraoperative Radiation Therapy. Medical Physics, 32, 3128-3131. http://dx.doi.org/10.1118/1.2044432

[2] Klink, C.D., Binnebosel, M., Holy, R., Neumann, U.P. and Junge, K. (2014) Influence of Intraoperative Radiotherapy (IORT) on Perioperative Outcome after Surgical Resection of Rectal Cancer. World Journal of Surgery, 38, 992-996. http://dx.doi.org/10.1007/s00268-013-2313-1

[3] Goubert, M. and Parent, L. (2015) Dosimetric Characterization of INTRABEAM((R)) Miniature Accelerator Flat and Surface Applicators for Dermatologic Applications. Physica Medica, 31, 224-232. http://dx.doi.org/10.1016/j.ejmp.2015.01.009

[4] Sperk, E., Astor, D., Keller, A., Welzel, G., Gerhardt, A., Tuschy, B., et al. (2014) A Cohort Analysis to Identify Eligible Patients for Intraoperative Radiotherapy (IORT) of Early Breast Cancer. Radiation Oncology, 9, 154. http://dx.doi.org/10.1186/1748-717X-9-154

[5] Sedlmayer, F., Reitsamer, R., Fussl, C., Ziegler, I., Zehentmayr, F., Deutschmann, H., et al. (2014) Boost IORT in Breast Cancer: Body of Evidence. International Journal of Breast Cancer, 2014, Article ID: 472516. http://dx.doi.org/10.1155/2014/472516

[6] Vaidya, J.S., Wenz, F., Bulsara, M., Tobias, J.S., Joseph, D.J., Keshtgar, M., et al. (2014) Risk-Adapted Targeted Intraoperative Radiotherapy versus Whole-Breast Radiotherapy for Breast Cancer: 5-Year Results for Local Control and Overall Survival from the TARGIT_A Randomised Trial. Lancet, 383, 603-613. http://dx.doi.org/10.1016/S0140-6736(13)61950-9

[7] Williams, N.R., Pigott, K.H., Brew-Graves, C. and Keshtgar, M.R. (2014) Intraoperative Radiotherapy for Breast Cancer. Gland Surgery, 3, 109-119.

[8] Eaton, D.J. and Duck, S. (2010) Dosimetry Measurements with an Intra-Operative X-Ray Device. Physics in Medicine and Biology, 55, N359-N369. http://dx.doi.org/10.1088/0031-9155/55/12/N02

[9] Armoogum, K.S., Parry, J.M., Souliman, S.K., Sutton, D.G. and Mackay, C.D. (2007) Functional Intercomparison of Intraoperative Radiotherapy Equipment—Photon Radiosurgery System. Radiation Oncology, 2, 11. http://dx.doi.org/10.1186/1748-717X-2-11

[10] Schneider, F., Clausen, S., Tholking, J., Wenz, F. and Abo-Madyan, Y. (2014) A Novel Approach for Superficial Intraoperative Radiotherapy (IORT) Using a $50 \mathrm{kV}$ X-Ray Source: A Technical and Case Report. Journal of Applied Clinical Medical Physics, 15, 4502.

[11] Ebert, M.A., Asad, A.H. and Siddiqui, S.A. (2009) Suitability of Radiochromic Films for Dosimetry of Very-Low Energy X-Rays. Journal of Applied Clinical Medical Physics, 10, 2957. http://dx.doi.org/10.1120/jacmp.v10i4.2957

[12] Soares, C., Drupieski, C., Wingert, B., Pritchett, G., Pagonis, V., O’brien, M., et al. (2006) Absorbed Dose Measurements of a Handheld $50 \mathrm{kVP}$ X-Ray Source in Water with Thermoluminescence Dosemeters. Radiation Protection Dosimetry, 120, 78-82. http://dx.doi.org/10.1093/rpd/nci622

[13] Eaton, D.J. (2012) Quality Assurance and Independent Dosimetry for an Intraoperative X-Ray Device. Medical Physics, 39, 6908-6920. http://dx.doi.org/10.1118/1.4761865 
[14] Ebert, M.A. and Carruthers, B. (2003) Dosimetric Characteristics of a Low-kV Intra-Operative X-Ray Source: Implications for Use in a Clinical Trial for Treatment of Low-Risk Breast Cancer. Medical Physics, 30, 2424-2431. http://dx.doi.org/10.1118/1.1595611

[15] Ebert, M.A., Carruthers, B., Lanzon, P.J., Haworth, A., Clarke, J., Caswell, N.M., et al. (2002) Dosimetry of a Low-kV Intra-Operative X-Ray Source Using Basic Analytical Beam Models. Australasian Physical Engineering Sciences in Medicine, 25, 119-123. http://dx.doi.org/10.1007/BF03178772 